\title{
La influencia de los grupos de interés en el comportamiento medioambiental de los directivos: el uso de estrategias indirectas
}

\author{
Javier Delgado Ceballos

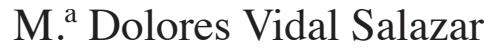 \\ Universidad de Granada
}

\section{Resumen}

Los grupos de interés han incrementado su preocupación por el medio ambiente empleando estrategias de presión directas e indirectas para modificar el comportamiento medioambiental de las empresas. Este trabajo exploratorio pretende aumentar el conocimiento sobre las estrategias de presión de los grupos de interés, y específicamente se centra en el análisis de la modificación del comportamiento medioambiental de los decisores ante la existencia de distintos niveles en la estrategia de comunicación dentro de las estrategias indirectas de presión empleando para ello una metodología de laboratorio. Esta investigación contribuye a la literatura de los grupos de interés al aumentar el conocimiento sobre cómo las estrategias de presión indirectas pueden variar la percepción de los decisores sobre asuntos medioambientales y cómo esta presión es más efectiva al emplear estrategias de comunicación de nivel alto.

Palabras clave: stakeholders, medio ambiente, estrategias indirectas de presión.

Clasificación JEL: M1, Q32.

\begin{abstract}
Stakeholders have increased their pressure on firms due to the rising concern about the natural environment in our society. This exploratory research aims enhancing our knowledge about stakeholders' pressure strategies, and specifically how different levels in the communication strategy of indirect actions by stakeholders alter decisors' environmental behavior. For that purpose, an experimental methodology, was carried to check if the use of an indirect strategy with a different situations are effective methods of pressure. This research contributes to the stakeholder literature showing the effect of indirect pressure strategies can have on managers' perception of environmental issues and that these strategies would be more effective if high communication strategies are employed.
\end{abstract}

Keywords: stakeholders, natural environment, indirect strategy of influence.

JEL classification: $M 1, Q 32$.

\section{Introducción}

Desde hace unas décadas el medio ambiente ha empezado tener una mayor relevancia en el mundo empresarial debido a la presión de la sociedad provocando que las empresas incluyan el medio ambiente en sus estrategias empresariales. Esta presión que realiza la sociedad se articula a través de los denominados grupos de interés o stakeholders (organizaciones no gubernamentales, reguladores, clientes, 
proveedores) que intentan conseguir que las empresas cambien sus estrategias y políticas medioambientales.

La literatura de los grupos de interés y medio ambiente ha centrado su atención en demostrar que los stakeholders pueden modificar el comportamiento medioambiental de aquellas empresas que son menos respetuosas con el mismo (Darnall et al., 2010; Delgado Ceballos et al., 2012; Rueda Manzanares et al., 2008; Surroca et al., 2013) e identificar qué stakeholders son los que principalmente afectan a la empresa (Buysse y Verbeke, 2003; Clarkson, 1995). En este sentido, la literatura ha resaltado la importancia de aquellos stakeholders que son considerados prominentes para los que toman las decisiones en las organizaciones (Mitchell et al., 1995) y que tienen un elevado poder respecto a las mismas (Agle et al., 1999). Asimismo, siguiendo la lógica de la Teoría de Recursos (Pfeffer y Salancik, 1978), se considera que aquellos stakeholders denominados primarios, es decir, que mantienen una relación económica directa con las empresas, tienen mayor capacidad para modificar el comportamiento de las mismas. De este modo, la literatura ha mostrado empíricamente la influencia de las presiones en materia medioambiental de aquellos stakeholders que son prominentes y tienen poder regulatorio o financiero sobre las empresas (Agle et al., 1999; Henriques y Sadorsky, 1999), considerándose al stakeholder regulador y a los accionistas como los agentes más influyentes en este tipo de decisiones (Henriques y Sadorsky, 1999). Por tanto, la capacidad de influencia de los stakeholders está relacionada con la prominencia del stakeholder (Mitchell et al., 1997), el poder del mismo (Agle et al., 1999) y el tipo de relación económica que tenga dicho stakeholder con la empresa (Frooman, 1999).

Además de los stakeholders primarios, existe otro tipo de stakeholders, denominados stakeholders secundarios, que no tienen ese poder tan decisorio sobre la empresa al no ser prominentes ni mantener una relación directa económica con las organizaciones (Clarkson, 1995); sin embargo, muchos de ellos presionan fuertemente a las empresas para que modifiquen su gestión medioambiental (Doh y Guay, 2006). Para lograr dicho propósito, los stakeholders secundarios suelen utilizar estrategias de presión directas e indirectas. Las primeras han recibido una mayor atención en la literatura y recogen aquellas acciones como protestas y campañas directas contra la actividad medioambiental de una determinada empresa. Las estrategias indirectas, en cambio, se producen «cuando un stakeholder trabaja a través de un aliado, teniendo el aliado la capacidad de manipular el flujo de recursos de la empresa. Así, aunque el stakeholder actué de forma indirecta, su aliado, por supuesto, actúa de forma directa» (Frooman, 1999: 198).

Por otro lado, algunos investigadores (Frooman, 1999; Frooman y Murell, 2005) subrayan la importancia de la estrategia de comunicación dentro de la estrategia indirecta de presión. Dicha estrategia se define como aquella dirigida a «informar al aliado sobre el comportamiento de la empresa y solicitarle con insistencia que se involucre para tomar una acción concreta» (Frooman, 1999: 199). Por tanto, se trata de una acción donde los stakeholders secundarios comunican a otros stakeholders capaces de afectar directamente a la empresa, sobre el comportamiento indeseable 
de ésta para que actúen en consecuencia gracias al poder de su influencia. En las estrategias indirectas juegan un papel fundamental las estrategias de comunicación donde se informa al potencial stakeholder aliado sobre el comportamiento de la organización donde se focaliza la presión. Por ejemplo, una organización no gubernamental (ONG) puede informar en su página web sobre una acción inapropiada de una empresa o puede aumentar la presión realizando anuncios en los medios de comunicación. El objetivo sería difundir información sobre el comportamiento inadecuado de dicha empresa con la intención de convencer a otros stakeholders más influyentes y conseguir su apoyo para, mediante una presión mayor, lograr el cambio de actuación por parte de la empresa. Sin embargo, escasa atención se ha dedicado comprender cómo el comportamiento de los decisores en las organizaciones puede cambiar dependiendo de la existencia de distintos niveles utilizados en la estrategia de comunicación dentro de la estrategia indirecta de presión de los stakeholders.

Este estudio exploratorio examina la influencia de las estrategias de presión indirectas en el comportamiento medioambiental de aquellos que son responsables de tomar decisiones en las organizaciones y analizar si los distintos niveles empleados por los stakeholders en la estrategia de comunicación pueden tener un impacto diferente en dicho comportamiento. Gracias al uso de una metodología de laboratorio, el juego del dictador, ha sido posible controlar la variable independiente. La investigación experimental (Kahneman et al., 1986) ha utilizado ya esta metodología en distintas investigaciones. El juego del dictador plantea un escenario donde dos individuos toman decisiones sobre la forma de compartir una cantidad fija de dinero. Uno de ello, el dictador, determina la división de dicha cantidad e impone la decisión al segundo sujeto, el recipiente. Mediante la aplicación de determinados tratamientos, el juego permite medir cómo una misma decisión puede variar al cambiar las circunstancias de partida a las que se enfrenta el tomador de decisiones.

En suma, las contribuciones de este trabajo están vinculadas a la relación entre las estrategias indirectas de presión de los stakeholders y la reacción de los agentes decisores de las organizaciones ante ellas. En concreto, las contribuciones son dos. En primer lugar, se aporta un mayor conocimiento sobre cómo los stakeholders secundarios pueden incrementar su influencia sobre las organizaciones mediante el uso de estrategias indirectas de presión. Segundo, el trabajo también incrementa conocimientos sobre cómo la aplicación de distintos niveles de intensidad en la estrategia de comunicación conlleva implicaciones y consecuencias sobre la influencia indirecta ejercida por estos stakeholders a los decisores.

El artículo procede de la siguiente manera: la siguiente sección se dirige al desarrollo teórico de los argumentos sobre las estrategias indirectas de presión; la tercera sección describe la muestra, el diseño del experimento y el procedimiento; la cuarta sección interpreta y discute los resultados del experimento; la última sección presenta las conclusiones del trabajo. 


\section{Teoría e hipótesis de trabajo}

\subsection{La capacidad de influencia de los stakeholders}

Desde el lanzamiento del libro seminal de Edward Freeman Strategic Management: A stakeholder approach (1984), se han publicado numerosos artículos sobre la influencia de los stakeholders en el comportamiento medioambiental de las organizaciones. Freeman definió el concepto de stakeholder como «cualquier grupo o individuo que puede afectar al desempeño de la empresa o es afectado por la misma en la consecución de sus objetivos» (Freeman, 1984: 46). Los investigadores han categorizado a los stakeholders en grupos para saber cuáles pueden afectar a las empresas (Clarkson, 1995; Freeman, 1984; Henriques y Sadorsky, 1999). Clarkson (1995) propuso clasificar a los stakeholders en primarios y secundarios. El primer grupo incluye a aquellos que mantienen una relación directa con la empresa -accionistas, gobiernos, trabajadores, clientes y proveedores- y que pueden influir notablemente en ella, siendo actores críticos para que la organización sobreviva. Los stakeholders secundarios -como las organizaciones no gubernamentales (ONGs) y los medios de comunicación- no tienen una relación directa con la empresa y son considerados como agentes de menor impacto respecto a la supervivencia de la empresa, por lo que su influencia se considerada menor que la que procede los stakeholders primarios.

Una de las líneas principales de investigación en la Teoría de los Stakeholder se ha centrado en analizar cómo las organizaciones actúan y reaccionan ante las presiones de los stakeholders respecto a cuestiones medioambientales (Berman et al., 1999; Carter, 2006; Hosmer y Kiewitz, 2005; Roome y Wijen, 2006). Estudios muestran que los responsables de tomar las decisiones medioambientales en las empresas modifican sus decisiones si reciben presiones de stakeholders primarios como los accionistas, trabajadores y proveedores. No obstante, otros estudios han analizado el poder de los stakeholders secundarios (Eesley y Lenox, 2006; Lounsbury, 2001; Sharma y Henriques, 2005). Por ejemplo, Sharma y Henriques (2005), en la industria forestal canadiense, y Lounsbury (2001) demostró cómo las presiones de la Asociación Estudiantil Student Environmental Action Coalition lograron influir positivamente para que las universidades adoptasen programas avanzados de reciclaje.

Específicamente, esta corriente de investigación también pretende aumentar la compresión sobre cómo los stakeholders secundarios consiguen influir a las organizaciones para mejorar su desempeño medioambiental. Los investigadores defienden que los stakeholders secundarios emplean diferentes métodos para influir en las empresas dependiendo de la relación económica existente entre ellos (Frooman, 1999; O'Connell et al., 2005; Sharma y Henriques, 2005) y de los medios (pathways) que el stakeholder posea para influir sobre la empresa (Frooman, 1999; Frooman y Murrell, 2005). 


\subsection{Estrategias indirectas de presión de los stakeholders}

Basándose en la Teoría de la Red, Rowley (1997) señala que no existe una relación únicamente dual entre la empresa y sus stakeholders, de forma que los stakeholders pueden tener una relación entre ellos mismos. Como consecuencia, su modelo «se mueve más allá del tradicional análisis dual y considera la influencia estructural y el impacto de los stakeholders que, aun no teniendo una relación directa con la empresa, se ven afectados por el comportamiento de la misma» (Rowley, 1997: 907). En esta misma línea, Frooman (1999) expande el tradicional modelo de centroradio (hub-and-spoke) que describe a la empresa como el centro de la rueda e indica que hay una relación dual entre ella y el los stakeholders, y lo modifica en un nuevo modelo donde los stakeholders de la empresa podrían tener una relación entre ellos y donde la empresa deja de ser el centro de la rueda. De este modo, un stakeholder aunque no tenga una relación directa de intercambio con la empresa, puede presionarla utilizando distintas formas de influencia, en concreto, estrategias directas e indirectas. Las estrategias directas se definen como «aquellas en las cuales los stakeholders manipulan el flujo de los recursos con la empresa» (Frooman, 1999: 198). Por ejemplo, las presiones directas ejercidas por los stakeholders serían: boicots (Garret, 1987, Pruitt et al., 1988), movilizaciones (Carmin y Balser, 2002; Rowley y Moldoveanu, 2003) o campañas de cartas (Smith y Cooper-Martin, 1997). Asimismo y siguiendo a Frooman (1999), los stakeholders también pueden presionar a las empresas a modificar sus comportamientos medioambiental mediante estrategias indirectas, las cuales se basan en el uso de aliados con influencia en la empresa. Este autor afirma que «cuando una empresa no tiene una relación de dependencia con los stakeholders, éstos no tienen capacidad de influir en ella, por lo que deben buscar un aliado del cual la empresa si sea dependiente» (Frooman y Murrell, 2005: 10). En otras palabras, en una estrategia indirecta, un stakeholder busca a un aliado para forzar el cambio en una determinada empresa haciendo que la influencia del aliado consiga modificar el comportamiento del directivo.

La literatura sobre los stakeholders subraya la importancia de las estrategias indirectas ya que éstas refuerzan el poder de los stakeholders secundarios sobre las empresas (Mitchell et al., 1997), todo ello gracias a las interacciones con stakeholder primarios con poder para influir sobre aquellos que toman las decisiones en las organizaciones (Clarkson, 1995, Freeman, 1984). Este razonamiento general sustentado por la literatura se considera puede ser trasladado al ámbito de las decisiones en materia medioambiental, defendiendo que el uso de una estrategia indirecta de presión puede modificar el comportamiento medioambiental de los decisores en una organización. En base a ello, se propone la siguiente hipótesis:

Hipótesis 1: El uso de una estrategia de presión indirecta incrementa la probabilidad de mejorar el comportamiento medioambiental del decisor en materia medioambiental. 


\subsection{Niveles en la estrategia de comunicación dentro de la estrategia indirecta de presión usada por los stakeholders}

En el uso de una estrategia indirecta es crucial para el stakeholder comunicar al aliado en qué consiste el comportamiento inadecuado de la empresa y cuáles deben ser las acciones futuras que el stakeholder aliado debe hacer para presionar directamente a la empresa (Frooman y Murell, 2005). Frooman (1999: 198) afirma que «las estrategias de comunicación son aquellas utilizadas por el stakeholder para informar al aliado potencial sobre (1) el comportamiento de la empresa, (2) por qué se considera que el comportamiento es inadecuado y (3) qué debería hacer el aliado». Pese a explicar la importancia de la estrategia de comunicación en las estrategias indirectas de presión, Frooman (1999) no tiene en cuenta los distintos niveles dentro de la estrategia de comunicación que los stakeholders pueden desarrollar para aumentar su influencia sobre las empresas.

Así, se considera que los stakeholders pueden usar distintos niveles de comunicación en la estrategia indirecta para variar el nivel de exposición pública de las empresas a las que pretenden condicionar y de aquellos que toman las decisiones. En el caso de problemas medioambientales, un stakeholder puede usar su capacidad de difundir a la sociedad información medioambiental sobre una empresa para favorecer o posicionarse en contra de la misma. Por ejemplo, un stakeholder puede generar información en su página web sobre actuaciones perjudiciales de una empresa a su entorno como hizo Greenpeace con un informe en su página web sobre la incineradora de Serín (Asturias) donde denunciaba las practicas realizadas por esta empresa respecto al tratamiento de los residuos y ofrecía distintas alternativas más ecológicas (Greenpeace, 2010a). Asimismo, un stakeholder puede incrementar el nivel de comunicación utilizando medios de mayor alcance, por ejemplo a través de campañas nacionales sobre el comportamiento indeseado de la empresa. Un ejemplo de este incremento en la presión comunicadora lo ofrece Frooman (1999) que describe como el Earth Island Institute emitió un video en las principales cadenas de televisión norteamericanas para pedir a los consumidores que boicotearan a la empresa Starkists, una firma dedicada al enlatado de atún denunciada por comprar a compañías que en el proceso de captura de este pescado acababan también con la vida de delfines. Esta campaña logró que Starkists modificase su comportamiento medioambiental. Un ejemplo más reciente ha sucedido en Argentina donde Greenpeace logró que la firma de pilas Energizer se hiciera cargo de la recogida y reciclaje de las pilas después de una intensa campaña publicitaria (Greenpeace, 2010b).

Así pues, los diferentes niveles en la estrategia de comunicación tienen distinto impacto en las decisiones relacionadas con el medio ambiente de las empresas. El poder de la estrategia de comunicación depende del nivel de exposición que el decisor dentro de la empresa sienta sobre sus decisiones. Por tanto, según consiga el stakeholder que la comunicación sea efectiva y aumente el nivel de exposición del decisor, mayor será su poder sobre el mismo y su capacidad para modificar su comportamiento. Por ello, se considera que una estrategia de presión indirecta con 
un nivel alto de comunicación sobre el comportamiento de aquellos que toman las decisiones en las organizaciones afectará más que en un nivel de comunicación bajo. En base a este razonamiento, planteamos la siguiente la hipótesis:

Hipótesis 2: Cuanto mayor sea el nivel de exposición de las decisiones percibido por los decisores en la estrategia de comunicación de los stakeholders, mayor será la probabilidad de modificar el comportamiento medioambiental de los mismos.

\section{Metodología}

\subsection{Diseño y funcionamiento del experimento}

Para analizar cómo el poder de las estrategias de presión de los stakeholders, principalmente los stakeholders secundarios, puede aumentar utilizando diferentes niveles en la estrategia de comunicación, se utiliza una metodología experimental siguiendo la propuesta de Frooman (1999), que afirma que este tipo de metodología puede ser la opción más idónea para analizar la efectividad de las estrategias de los stakeholders. El uso de experimentos para extraer conclusiones aplicables al entorno empresarial del mundo real ha encontrado un fuerte apoyo en la literatura (Arnott y O’Donnell, 2008; Chung et al., 2004; Reijers et al., 2011) ya que «los experimentos permiten crear ambientes similares a sus análogos mundo real al emular las condiciones del mundo real en el laboratorio» (Kim et al., 2002: 216).

En nuestro experimento se aplica el juego del dictador. A fin de exponer en qué consiste, primero se explica el juego del ultimátum que representa la situación más simple de negociación: dada una cantidad inicial, el jugador 1 realiza una oferta al jugador 2: $(\mathrm{x} 1, \mathrm{x} 2)$. Si éste acepta ambos jugadores se quedan con el resultado de esta propuesta $(\mathrm{x} 1, \mathrm{x} 2)$, en caso contrario ambos jugadores se quedan con nada ( 0 , 0 ). De esta forma, siempre que $x 2 \geq 0$, el jugador 2 siempre aceptará la oferta. Así, el equilibrio de Nash predice que el jugador 1 realizará una oferta donde $\mathrm{x} 1 \leq \mathrm{P}$ y x $2 \geq 0$. El juego del dictador es idéntico al del ultimátum con la diferencia que el jugador 2 no tiene poder, teniendo que aceptar cualquier oferta que le realice el jugador 1 . No teniendo poder alguno el jugador 2, la predicción es que el jugador 1 siempre llevará a cabo la oferta más ventajosa para él, que sería además la más desventajosa para el jugador 2. Sin embargo, la literatura del juego del dictador ha mostrado cómo los dictadores normalmente donan entre el 20-30 por 100 del presupuesto al jugador 2 (Eckel y Grossman, 1998; Gurven, 2004). 


\subsection{Participantes}

Numerosos trabajos han utilizado estudiantes como muestra para sus estudios (Stark et al., 2007, Turban y Greening, 1997). Para elaborar este trabajo, participaron en el experimento 112 estudiantes que cursaban el módulo de dirección estratégica dentro un Máster Oficial de la Universidad de Granada (España). La mayoría de los estudiantes habían tenido alguna experiencia laboral desarrollando trabajos a tiempo completo (70 por 100) y estaban acabando ese año sus estudios académicos ( 80 por 100). En este caso, las características de la muestra (estudiantes con un conocimiento avanzado en gestión de empresas, alguna experiencia profesional y alta motivación) constituyen una buena aproximación al comportamiento de aquellos que toman las decisiones en un contexto profesional.

\subsection{Instrucciones del experimento}

Los participantes en el experimento se encontraban con una serie de instrucciones escritas en sus mesas y contaban también con la disponibilidad de varios tutores para consultar dudas y garantizar así que todos los participantes entendiesen claramente las tareas que tendrían que realizar. Antes de iniciar el experimento, las instrucciones fueron expuestas en voz alta a todo el colectivo participante. La única parte que no se expuso públicamente fue el apartado final de las instrucciones ya que éste difería dependiendo de cada uno de los distintos tratamientos utilizados en el experimento. Las instrucciones explicaban que se había producido un aumento de la preocupación de la sociedad sobre la conservación del Amazonas, algo que se evidenciaba por el elevado número de asociaciones creadas para proteger el Amazonas de la especulación. A los participantes se les entregaban diez monedas de un euro de forma que podían dividir la cantidad entre ellos mismos y una asociación creada para dicho fin: la ONG «Amazonia Rainforest Heroes», que lleva trabajando por la conservación de los bosques desde 1985. A los participantes se les informaba en las instrucciones de que cada euro donado lograría salvar cuatro árboles. Se explicaba claramente la finalidad de la donación con el fin de eliminar suspicacias respecto a los fines a los que se dedicaba el dinero, lo cual implica que las instrucciones animaban al decisor a dotar al máximo la cantidad de dinero donada a la ONG. Finalmente, resaltar que los participantes se quedaban con la cantidad no donada y que no recibieron ningún tipo de coacción en sus decisiones, adoptando caca cual la donación que considerase más apropiada.

\subsection{Medidas y tratamientos}

En el experimento se desarrollaron tres tratamientos para testar la efectividad de nuestras hipótesis. El modelo 1, denominado sin estrategia indirecta, fue el modelo 
base y el que se utilizó para llevar a cabo las comparaciones. En este modelo, las donaciones que se realizaban eran confidenciales y no se hacían públicas. De esta manera, en las instrucciones de este modelo se informaba a los participantes que nadie conocería los resultados del experimento y que, por tanto, no se harían públicas las cantidades donadas por cada uno de ellos. Destacar que este modelo no recoge ningún tipo de no existe presión de ningún tipo hacia los decisores ya que éstos tienen la creencia de que nadie conocerá cuál ha sido su elección. En el modelo 2, denominado estrategia indirecta con un bajo nivel de comunicación, los tutores informaron a los participantes que sus nombres aparecerían en una lista que indicaría sus donaciones clasificadas desde la cantidad donada más baja a la más alta junto con sus nombres completos. Aquellos que hubieran donado menos se encontrarían encabezando la lista. La lista se expondría en la puerta de la clase donde se llevó a cabo el experimento durante una semana. Además, en las instrucciones se especificaba a los alumnos que si la lista se destruía, se volvería a reemplazar y estaría expuesta incluyendo el periodo de tiempo perdido. En este modelo, se quiso medir si había un incremento de las probabilidades de mejora del comportamiento de los decisores cuando éstos se ven expuestos a una presión por parte de la actuación de un stakeholder capaz de crear opinión en terceros considerados importantes para el decisor, al hacer pública su actuación utilizando una estrategia indirecta con un nivel moderado de comunicación. En el modelo 3, denominado estrategia indirecta con un alto nivel de comunicación, las instrucciones indicaban que una vez que el experimento concluyera se llevaría a cabo un anuncio público y presencial en la propia clase de las donaciones realizadas por cada participante. En este último modelo, se pretendía medir si el comportamiento de los decisores podía condicionarse más por aumentar la presión indirecta al incrementarse el impacto de la comunicación a realizar.

Por último, la premisa básica de la Teoría de los Stakeholders es el enfrentamiento entre los stakeholders y las empresas. Aplicada al ámbito de gestión medioambiental, los estudios mantienen que los stakeholders centran su presión en aquellas organizaciones que consideran no llevan a cabo las acciones medioambientales apropiadas mientras que si los stakeholders consideran esa gestión idónea, no realizan ninguna presión sobre la empresa, al contrario, incluso pueden ofrecer algún tipo de reconocimiento por dicho comportamiento. Siguiendo este mismo razonamiento, en el experimento, dependiendo de la cantidad donada, se identificaron aquellos sujetos que tenían menor compromiso con el medio ambiente, siendo la población de los tres los modelos dividida en dos grupos. Con esta subdivisión, se quería comprobar si la media de donaciones de aquellos sujetos menos comprometidos con el medio ambiente era diferente dependiendo del nivel de exposición a la que se veía expuesto según la estrategia de comunicación aplicada en el experimento. No interesaba el grupo de aquellos participantes más concienciados medioambientalmente ya que se presuponía que estos sujetos iban a actuar correctamente tanto si había como si no había estrategia indirecta o una mayor o menor estrategia de comunicación dentro de ella. Se utilizaron distribuciones acumulativas para analizar las donaciones de manera que se describe la probabilidad que una variable x obtenga un valor menor o igual 
a un número x. El primer grupo (Grupo 1) estaba formado por un 50 por 100 de los participantes que realizaban las donaciones más altas, de manera que se asumía que este grupo tendría un mayor compromiso con el medioambiente. El segundo grupo (Grupo 2) estaba formado por aquellos participantes que donaban menos dinero, de manera que se asumía que este grupo tendría un menor compromiso medioambiental. De esta forma, la muestra final estaba formada por 54 sujetos, todos con bajo compromiso medioambiental, divididos en tres grupos que eran sometidos a estrategias indirectas de presión con distintos niveles de intensidad en la comunicación.

\section{Análisis y resultados}

En el experimento, los estudiantes donaron un total de 821 euros de la cantidad total repartida que fueron 1.130 euros (10 euros por estudiante) y se quedaron con un total de 309 euros. De esta forma, los participantes ganaron una media de 2,73 euros. La participación en el experimento era voluntaria y los participantes eran conscientes de que las donaciones las recibiría realmente la ONG.

Como muestra de los resultados, resaltar que el 38 por 100 de los participantes del modelo base donaron todo el presupuesto, los 10 euros. El resultado es consistente si se compara con otros juegos del dictador donde se da información sobre los receptores de los fondos. Por ejemplo, en un experimento similar desarrollado por Burnham (2003), el 25 por 100 de los participantes donaron la mitad del presupuesto en un juego del dictador. De manera sorprendente, en un estudio realizado por Eckel y Grossman (1996) sólo el 10 por 100 de los participantes donaron todo a la Cruz Roja que fue el receptor en este caso. Sin embargo, el 70 por 100 de los participantes donaron toda la cantidad cuando se les informó que el dinero iba a ser destinado a los pobres en África. Por lo tanto, los resultados muestran que el mecanismo del experimento fue creíble. Esto explica por qué los participantes donaron una cantidad media significativa en el tratamiento base.

Para testar la primera Hipótesis, se realizó una comparación de medias utilizando el análisis ANOVA en el programa SPSS 16 (Cuadro 1). En base a los resultados obtenidos, la hipótesis 1 , que predice que los decisores adoptarán un comportamiento más respetuoso con el medio ambiente cuando se enfrentan a una estrategia indirecta por parte de los stakeholders, fue aceptada. Este resultado implica que el tratamiento de estrategia indirecta puede ayudar a modificar el comportamiento irrespetuoso con el medio ambiente. 


\section{CUADRO 1}

RESULTADOS ANÁLISIS ANOVA DE COMPARACIÓN DE MEDIAS

\begin{tabular}{|l|c|c|}
\hline \multicolumn{1}{|c|}{ Tratamiento } & Media & ANOVA F \\
\hline Baseline & $4 \quad(\mathrm{n}=18)$ & $9,723 * * *$ \\
\hline Estrategia indirecta de presión & $5,86(\mathrm{n}=36)$ & \\
\hline
\end{tabular}

NOTA: Significativo en los siguientes niveles $† 0,10 ; * 0,05 ; * * 0,01 ; * * * 0,001 . \mathrm{n}=54$.

Por otro lado, el trabajo pretendía igualmente testar si el nivel de la estrategia de comunicación podía afectar al comportamiento medioambiental del decisor. Para probar la segunda hipótesis se ha utilizado el Test Post-Soc Sheffe, que identifica diferencias significativas entre el nivel base y ambos niveles de comunicación.

\section{CUADRO 2}

ANÁLISIS DE POST HOC SHEFFE

\begin{tabular}{|c|c|c|}
\hline Estrategia $(\mathbf{I})$ & Estrategia $(\mathbf{J})$ & Diferencia de medias $(\mathbf{I}-\mathbf{J})$ \\
\hline \multirow{2}{*}{ Baseline } & Nivel bajo de comunicación & $-1,000 \quad(0,653)$ \\
\cline { 2 - 3 } & Nivel alto de comunicación & $-2,722 * * *(0,653)$ \\
\hline \multirow{2}{*}{ Nivel bajo de comunicación } & Baseline & $-1,000 * *(0,653)$ \\
\cline { 2 - 3 } & Nivel alto de comunicación & $-1,722^{* *}(0,653)$ \\
\hline \multirow{2}{*}{ Nivel alto de comunicación } & Baseline & $2,722 * * *(0,653)$ \\
\cline { 2 - 3 } & Nivel bajo de comunicación & $1,722^{* *}(0,653)$ \\
\hline
\end{tabular}

NOTA: Significativo en los siguientes niveles: $\uparrow 0,10 ; * 0,05 ; * * 0,01 ; * * * 0,001 . \mathrm{n}=54$.

La Hipótesis 2, que intenta predecir modificaciones en los comportamientos de los tomadores de decisiones según se someterían a diferentes modelos que reproducían distintas estrategias de presión según diferentes niveles de comunicación, fue también aceptada. De acuerdo con el resultado del Test Sheffe mostrado en el Cuadro 2, el modelo estrategia indirecta con un alto nivel de comunicación es más dominante que el modelo estrategia indirecta con un bajo nivel de comunicación $(\mathrm{p}=0,038)$ y que el de nivel base $(\mathrm{p}=0,001)$. De estos resultados, se puede concluir que cuando la estrategia de comunicación alcanza a más gente o se expone utilizando medios más efectivos, los decisores reciben más presión y varían su percepción sobre las cuestiones medioambientales, de tal manera que el cambio en el comportamiento que va a ser expuesto es mayor. En contra de nuestras expectativas, los resultados mostraron que no existe una diferencia significativa entre el modelo $1 \mathrm{y}$ el modelo base. Este resultado podría deberse, por una parte, al alto nivel de valores medioambientales de los decisores que formaban la muestra utilizada y, por otra, a que los decisores no modifican realmente su comportamiento medioambiental si no perciben un nivel de presión elevado. 
En resumen, los resultados del experimento realizado con alumnos para predecir las modificaciones en el comportamiento medioambiental en decisores al verse expuestos a estrategias indirectas de presión y dentro de ellas, a distintos niveles de intensidad de la presión en base diferentes estrategias de comunicación a la hora de exponer públicamente los resultados, indican que las estrategias indirectas son efectivas a la hora de modificar los comportamientos medioambientales y que cuando la exposición de dichos comportamientos es pública y efectiva gracias a estrategias de comunicación consideradas de alta intensidad, tiene un mayor impacto en el cambio de comportamientos en esta materia.

\section{Discusión y conclusiones}

Este estudio aborda la cuestión de cómo los decisores de las organizaciones reaccionan a la presión de los stakeholders, siendo el punto de partida de la investigación el trabajo de Frooman (1999) que indica la necesidad de analizar el impacto de las estrategias de los stakeholders sobre el comportamiento medioambiental de los directivos. Concretamente, este trabajo se centra en las estrategias indirectas de presión, siendo nuestro objetivo mejorar la comprensión de cómo los decisores menos comprometidos con el medio ambiente reaccionan frente a la utilización de estrategias indirectas por parte de los stakeholders secundarios. Además, se testa cómo la existencia de diferentes niveles de comunicación en las estrategias indirectas juega un papel clave en dichas decisiones.

El trabajo contribuye a la literatura sobre gestión de los stakeholders y medio ambiente de diferentes formas. En primer lugar, el estudio concluye que las estrategias indirectas modifican las decisiones medioambientales de los responsables empresariales, especialmente si los stakeholders utilizan una estrategia de comunicación alta. Como consecuencia, los resultados del estudio muestran que los stakeholders secundarios, pese a no ser fundamentales para los intereses de los decisores, pueden influir notablemente en éstos usando una estrategia indirecta de presión que logra incrementar su poder. Asimismo, los stakeholders primarios y los prominentes (el gobierno) también pueden utilizar estas estrategias indirectas para reforzar su poder ya que son más baratas y más fáciles de implantar que las estrategias directas o aquellas medidas más conflictivas. De esta forma, un stakeholder puede incrementar su capacidad de influencia sobre los decisores informando a otros stakeholders sobre el comportamiento inadecuado de la empresa, ganando así aliados en otros grupos de interés y logrando incrementar su poder en la relación con la empresa sobre la que desean influir.

En segundo lugar, también se contribuye al conocimiento sobre los stakeholders demostrando que el impacto de las estrategias indirectas está relacionado con el nivel de exposición de las decisiones adoptadas por los responsables. Esta afirmación se basa en los efectos logrados al exponer a los decisores a distintos niveles de comunicación en la estrategia indirecta. En la actualidad, las estrategias indirectas de los 
stakeholders relacionadas con el medio ambiente están mayoritariamente basadas en la publicación de informes medioambientales de las empresas sobre las que desean influir en páginas web por otros stakeholders. Por ejemplo, algunos gobiernos y algunas organizaciones internacionales han desarrollado en las últimas décadas listas detalladas de empresas contaminantes en muchos países como el Toxics Release Inventory en los Estados Unidos o el Pollutant Release and Transfer Register europeo. En este caso, se trata de estrategias de comunicación consideradas de bajo impacto lo que conlleva que los directivos se consideren sólo moderadamente expuestos a la opinión pública. Así, la publicación de esta información puede que no sea suficiente para modificar el comportamiento medioambiental de los directivos ya que la información facilitada en la red no alcanza a la sociedad en general. Se necesitaría incrementar la difusión de esta información mediante estrategias de comunicación de mayor intensidad para alcanzar a la sociedad en general. Si se extrapolan los resultados de la presente investigación al mundo de la empresa se podría afirmar que al aumentar la intensidad de comunicación usando medios o canales de comunicación de mayor impacto, incluso siendo locales, se logra una mayor influencia sobre el comportamiento medioambiental al incrementar el nivel de exposición de las decisiones de los directivos y, de esta forma, el nivel de exposición de la empresa y de sus comportamientos. Ese incremento en el nivel de comunicación se podría traducir en un mayor desempeño medioambiental, especialmente en aquellas empresas con actuaciones poco respetuosas con el medio ambiente o empresas que cuentan con directivos que tienen menor sensibilidad hacia cuestiones medioambientalmente.

En tercer lugar, este artículo contribuye a la literatura debido a la metodología que utilizada para testar cómo las diferentes estrategias indirectas pueden influir en el comportamiento medioambiental de los decisores al tener en cuenta sus acciones y no sus percepciones. Estudios previos (Murillo Luna et al., 2008) sobre cómo las presiones de los stakeholders afectan a los directivos han utilizado encuestas y estudios de caso. Una excepción al uso de los análisis tradicionales sería el trabajo de Eesely y Lenox (2006) donde se medían las acciones de los directivos en vez de sus percepciones mediante datos secundarios. En el caso de este trabajo, creemos que puede ayudar a incrementar el uso de este tipo de metodología cuando se tenga como objetivo medir una serie de constructos que son difícilmente cuantificables con datos primarios o secundarios.

Como aportación a la gestión, es importante destacar que los responsables de tomar decisiones en materia medioambiental de las organizaciones (ya sean empresas, instituciones, organismos...) deben conocer cuáles son sus stakeholders, cuáles son sus estrategias así como su capacidad de influencia para poder planificar y actuar estratégicamente a este respecto. Un mayor conocimiento del comportamiento de los agentes de su entorno les permitirá anticiparse y actuar en consecuencia. Además, dicho conocimiento incentivará a las empresas a incrementar la información medioambiental que proporcionan para lograr mejorar su reputación medioambiental y las relaciones con sus stakeholders al legitimar las acciones de la empresa mediante la información proporcionada. 
Pese a las aportaciones realizadas, el estudio presenta dos limitaciones principales. La primera, es la utilización de estudiantes como aproximación a la realidad de las organizaciones y de sus decisiones con respecto al medioambiente. Aunque el nivel de conocimiento sobre el mundo empresarial de nuestros participantes es apropiado para lograr eficacia en los tratamientos planteados en la investigación, la literatura experimental (Cooper et al., 1999; Montmarquette et al., 2004) reconoce que los directivos podrían elegir opciones estratégicas diferentes. La segunda limitación reside en que el análisis se centra únicamente en un cierto tipo de presión ejercida por los stakeholders a los estudiantes para modificar sus decisiones medioambientales. Reconocemos que en el mundo empresarial, los responsables medioambientales también están influenciados por otros factores como las presiones de otros stakeholders o problemas internos de sus organizaciones. Por ejemplo, en el experimento, un participante que dona al 100 por 100 del dinero a una ONG es percibida de manera muy positiva por el resto de los stakeholders. No obstante, en una empresa, si el directivo donase el 100 por 100 de los beneficios extraordinarios de un ejercicioa acciones sociales, probablemente algunos stakeholders (los propietarios) tendrían una percepción negativa de la decisión y del desempeño del directivo.

En todo caso, tal y como se argumenta en la literatura sobre stakeholders, son necesarios más estudios que aumenten el conocimiento sobre las distintas estrategias de presión ejercidas por los grupo de interés y su influencia y efectividad sobre las empresas. Como línea de investigación en este sentido, sería interesante analizar cómo los directivos y las empresas perciben y reaccionan ante la publicación y exposición pública de datos medioambientales por parte de los gobiernos pero no desde un punto de vista financiero sino desde el punto de vista de gestión del directivo frente a la exposición pública de dicha información. Otra línea de investigación podría ser analizar el papel de las ONG y las redes sociales para difundir los datos de estos registros a la sociedad y testar en qué medida afectaría esto a la estrategia medioambiental de las empresas y conocer el impacto interno sobre las organizaciones afectadas, sobre todo si éstas varían los medios, canales o estrategias de comunicación utilizadas.

\section{Referencias bibliográficas}

[1] AGLE, R. B.; MITCHELL, R. K. y SONNENFELD, J. A. (1999): «Who matters to CEOs? An investigation of stakeholder attributes and salience, corporate performance, and CEO values», Academy of Management Journal, vol. 42, n. . . 5, pp. 507-525.

[2] ARNOTT, D. y O'DONNELL, P. (2008): «A note on an experimental study of DSS and forecasting exponential growth», Decision Support Systems, núm. 45, pp. 180-186.

[3] BERMAN, S. L.; WICKS, A. C.; KOTHA, S. y JONES, T. M. (1999): «Does stakeholder orientation matter? The relationship between stakeholder management models and firm financial performance», Academy of Management Journal, vol. 42, n. . 5, pp.488506. 
[4] BURNHAM, T.C. (2003): «Engineering altruism: a theoretical and experimental investigation of anonymity and gift giving», Journal of Economics Behavior and Organization, vol. 50, nº. 1, pp. 133-144.

[5] BUYSSE, K. y VERBEKE, A. (2003): «Proactive environmental strategies: A stakeholder management perspective», Strategic Management Journal, vol. 24, n. ${ }^{\circ}$ 5, pp. 453-470.

[6] CARMIN, J. y BALSER, D. B. (2002): «Selecting repertoires of action in environmental movement organizations», Organization and Environment, vol. 15, pp. 365-388.

[7] CARTER, S. M. (2006): «The interaction of top management group, stakeholder, and situational factors on certain corporate reputation management activities», Journal of Management Studies, vol. 43, n. ${ }^{\circ}$ 5, pp.1145-1176.

[8] CHUNG, W.; ZHANG, Y.; HUANG, Z.; WANG, G.; ONG, T. y CHEN, H.(2004): «Internet searching and browsing in a multilingual world: an experiment on the Chinese business intelligence portal (CBizPort)», Journal of the American Society for Information Science and Technology, vol. 55, n. ${ }^{\circ}$ 9, pp. 818-831.

[9] CLARKSON, M. B. E. (1995): «A stakeholder framework for analyzing and evaluating corporate social performance», Academy of Management Review, vol. 20, n. ${ }^{\circ}$ 1, pp. 65-91.

[10] COOPER, D. J.; KAGEL, J. H.; LO, W. y GU, Q. L. (1999): «Gaming against managers in incentive systems: Experimental results with Chinese students and Chinese managers», American Economics Review, vol. 89, n. ${ }^{\circ}$ 4, pp. 781-804.

[11] DARNALL, N.; HENRIQUES, I. y SADORSKY, P. (2010): «Adopting proactive environmental strategy: the influence of stakeholders and firm size», Journal of Management Studies, vol. 47, n. ${ }^{\circ}$ 6, pp. 1072-1094.

[12] DELGADO CEBALLOS, J.; ARAGÓN CORREA, J.; ORTIZ DE MANDOJANA, N. y RUEDA MANZANARES, A. (2012): «The effect of internal barriers on the connection between stakeholder integration and proactive environmental strategies», Journal of Business Ethics, vol. 107, n.3, pp.281-293..

[13] DOH, J. y GUAY, T. R. (2006): «Corporate Social Responsibility, Public Policy, and NGO Activism in Europe and the United States: An Institutional-Stakeholder Perspective», Journal of Management Studies, vol. 43, n. ${ }^{\circ}$ 1, pp. 47-73.

[14] ECKEL, C. y GROSSMAN, P. (1996): «Altruism in anonymous dictator games», $G a-$ mes and Economic Behavior, vol. 16, n. ${ }^{\circ}$ 2, pp. 181-191.

[15] EESLEY, C. y LENOX, M. J. (2006): «Firm responses to secondary stakeholder action», Strategic Management Journal, vol. 27, n. ${ }^{\circ}$ 8, pp. 765-781.

[16] FINEMAN, S. (1996): «Emotional subtexts in corporate greening», Organization Studies, vol. 17, n. . 3, pp. 479-500.

[17] FORSYTHE, R.; HOROWITZ, J. L.; SAVIN, N. E. y SEFTON, M. (1994): «Fairness in simple bargaining experiments», Games and Economic Behavior, vol. 6, pp. 347369.

[18] FREEMAN, R. E. (1984): Strategic Management: A Stakeholder Approach, Pitman/ Ballinger, Boston.

[19] FROOMAN, J. (1999): «Stakeholder influence strategies», Academy of Management Journal, vol. 24, n. ${ }^{\circ}$ 2, pp. 191-205.

[20] FROOMAN, J. y MURRELL, A.J. (2005): «Stakeholder Influence Strategies: The Roles of Structural and Demographic Determinants», Business \& Society, vol. 44, n. ${ }^{\circ}$, pp. 3-31. 
[21] GARRET, D. E. (1987): «The effectiveness of marketing policy boycotts», Journal of Marketing, vol. 51, pp. 46-57.

[22] GREENPEACE (2010a): http://www.greenpeace.org/raw/content/espana/reports/ 100731.pdf, visto el día 15 de febrero 2013.

[23] GREENPEACE (2010b): http://www.greenpeace.org/argentina/prensarss/energizerresponsable-pilas, visto el día 5 de julio 2013.

[24] GURVEN, M. (2004): «Economic games among Amazonian Tsimane: Exploring the roles of market access, costs of giving, and cooperation on pro-social game behavior», Experimental Economics, vol. 7, n. ${ }^{\circ}$ 1, pp. 5-24.

[25] HENRIQUES, I. y SADORSKY, P. (1999): «The relationship between environmental commitment and managerial perceptions of stakeholder importance», Academy of Management Journal, vol. 42, n. ${ }^{\circ}$ 1, pp. 87-99.

[26] HENRIQUES, I. y SHARMA, S. (2005): «Pathways of Stakeholder Influence in the Canadian Forestry Industry», Business Strategy and the Environment, vol. 14, n. ${ }^{\circ}$ 6, pp. 384-398.

[27] HOSMER, L. T. y KIEWITZ, C. K. (2005): «Organizational justice: A behavioral science concept with critical implications for business ethics and stakeholder theory», Business Ethics Quarterly, vol. 15, n. ${ }^{\circ}$ 1, 67-91.

[28] KAHNEMAN, D.; KNETSCH, J. y THALER, R.H. (1986): «Fairness and the assumptions of economics», Journal of Business, vol. 59, n. ${ }^{\circ} 4$, pp. 285-300.

[29] KIM, B.; BARUA, A. y WHINSTON, A. B. (2002): «Virtual field experiments for a digital economy: a new research methodology for exploring an information economy», Decision Support Systems, n. 32, pp. 215-231.

[30] MITCHELL, R. K.; AGLE, R. B. y WOOD, D. J. (1997): «Toward a theory of stakeholder identification and salience: Defining the principle of who and what really counts», Academy of Management Review, vol. 22, n. ${ }^{\circ} 4$, pp. 853-886.

[31] MONTMARQUETTE, C.; RULLIÈRRE, J. L.; VILLEVAL, M. C. y ZEILIGER, R. (2004): «Redesigning teams and incentives in a merger: An experiment with managers and students», Management Science, vol. 50, n. ${ }^{\circ}$ 10, pp. 1379-1389.

[32] MURILLO-LUNA, J. L.; GARCÉS-AYERBE, C. y RIVERA-TORRES, P. (2008): «Why do patterns of environmental response differ? A stakeholders' pressure approach», Strategic Management Journal, vol. 29, n. . 11, pp. 1225-1240.

[33] LOUNSBURY, M. (2001): «Institutional sources of practice variation: staffing college and university recycling programs», Administrative Science Quarterly, vol. 46, pp. 29-56.

[34] O'CONNELL, L. C.; STEPHENS, C.; BETZ, M.; SHEPARD, J. y HENDRY, J. (2005): «An organizational field approach to corporate rationality: the role of stakeholder activism», Business Ethics Quarterly, vol. 15, n. ${ }^{\circ}$ 1, pp. 93-111.

[35] PFEFFER, J. y SALANCIK, G. (1978): The external control of organizations: A resource dependence perspective, Harper \& Row, New York.

[36] PRUITT, S. W.; WEI, K. C. y WHITE, R. E. (1988): «The impact of union-sponsored boycotts on the stock prices of target firms», Journal of Labor Research, vol. 9, n. ${ }^{\circ}$, pp. 285-289.

[37] REIJERS, H. A.; FREYTAGB, T.; MENDLINGC, J. y ECKLEDERB, A. (2011): «Syntax highlighting in business process models», Decision Support Systems, vol. 51, n. ${ }^{\circ}$, pp. 339-349.

[38] ROOME, N. y WIJEN, F. (2006): «Stakeholder Power and Organizational Learning 
in Corporate Environmental Management», Organization Studies, vol. 27, n. ${ }^{\circ}$ 2, pp. 235-263.

[39] ROWLEY, T. J. (1997): «Moving beyond dyadic ties: A network theory of stakeholder influences», Academy of Management Journal, vol. 22, n. . 4, pp. 887-910.

[40] ROWLEY, T. J. y MOLDOVEANU, M. (2003): «When will stakeholder groups act? An interest and identity-based model of stakeholder group mobilization», Academy of Management Review, vol. 28, n. ${ }^{\circ}$ 2, pp. 204-219.

[41] RUEDA MANZANARES, A.; ARAGÓN CORREA, J. A. y SHARMA, S. (2008): «The Influence of Stakeholders on the Environmental Strategy of Service Firms: The Moderating Effects of Complexity, Uncertainty and Munificence», British Journal of Management, vol. 19, n. ${ }^{\circ}$ 2, pp. 185-203.

[42] SHARMA, S. y HENRIQUES, I. (2005): «Stakeholders, environment and society: multiple perspectives, emerging consensus», en Sharma, S. y M. Starik (eds.), New Perspectives on Research in Corporate Sustainability: Stakeholders, Environment and Society, Edward Elgar Academic Publishing, Northampton, MA.

[43] SMITH, N. C. y COOPER-MARTIN, E. (1997): «Ethics and target marketing: The role of product harm and consumer vulnerability», Journal of Marketing, vol. 61, n. . 3, pp. $1-20$.

[44] SURROCA, J.; TRIBÓ, J. A. y ZAHRA, S. (2013): «Stakeholder Pressure on MNEs and the Transfer of Socially Irresponsible Practices to Subsidiaries», Academy of Management Journal, vol. 56, n. . 2, pp. 549-572.

[45] STARK, E. M.; SHAW, J. D. y DUFFY, M. K. (2007): «Preference for group work, winning orientation, and social loafing behavior in groups», Group \& Organization Management, vol. 32, n. ${ }^{\circ}$ 6, pp. 699-723.

[46] TURBAN, D. B. y GREENING. D. W. (1997): «Corporate social performance and organizational attractiveness to prospective employees», Academy of Management Journal, vol. 40, n. ${ }^{\circ}$ 3, pp. 658-672. 